\title{
High granularity scanner for MPGD based photon detectors
}

\author{
Gergő HAMAR* \\ Wigner Research Centre for Physics, Budapest \\ E-mail: hamar.gergo@wigner.mta.hu \\ Dezső VARGA \\ Wigner Research Centre for Physics, Budapest \\ E-mail: varga.dezso@wigner.mta.hu
}

\begin{abstract}
Gaseous detectors can be made sensitive to photons, and become excellent choice for applications such as Cherenkov radiation imaging for particle identification. Micropattern Gaseous Detector (MPGD) technologies opened new ways to photon detection, where the possibility for reduced ion feedback, better timing and the suppression of non-photon signals are factors of improved performance. On the other hand the microstructure of an MPGD renders the photo-electron emission, transfer and subsequent detection to be a very complex process.

We have developed a high resolution UV photon scanner, where single photon-electron response measures local detection efficiency and gas gain with position resolution better than 100 microns. Studies on Thick GEM based photon detectors proved the existence of inefficient symmetry points, and shed light on hole-gain structure and microscale variance. In fact practically all MPGD detectors, even if not designed for photon detection in the first place, can be made sensitive, and thus be explored, by the scanning system. Measurement of the microstructure of the charge transport can lead to a better understanding of the detection mechanisms, and help in optimization of various MPGD, especially for Cherenkov detectors.

The presentation will focus on details of the critical parts of such a system; and recent results on TGEM microstructure with its dependence on the applied micropattern configurations.
\end{abstract}

Technology and Instrumentation in Particle Physics 2014

2-6 June, 2014

Amsterdam, the Netherlands

\footnotetext{
* Speaker.
} 


\section{Introduction}

In modern particle physics the issue of precise particle identification in high momentum arose the need for large area Cherenkov detectors. Following the work done in RD26 excellent MWPC based gaseous photon-detectors have been constructed, however several difficulties are limiting the performance (timing, photon feedback, ion backflow, gain uniformity, ...). Novel micropattern gaseous detectors [1] gave a new rise for gaseous tracker and trigger devices, and Cherenkov detectors as well [2].

While in PHENIX HBD [3] triple GEM [4] foils were used for threshold Cherenkov detectors, for ring imaging application in VHMPID R\&D [5, 6] and in COMPASS RICH1 Upgrade [7] projects TGEM [8] based detectors were considered.

Advances from the new technology can be seen in timing, suppressed ion backflow, elimination of feedback photons, and possibility to suppress MIP signals. On the contrary holes cannot be covered with $C s I$, and due to the micropatterns they have highly inhomogeneous field structure. Optimization of geometrical parameters (hole diameter, pitch, thickness and rim) together with the electrical settings defines a large parameter-space.

We have developed a system to get insight into the previous processes on the scale of the micropattern geometry. This paper describes the basic setup and its recently upgraded data acquisition system, as well as results from TGEM studies and preliminary plots taken with standard GEM foils.

\section{The Leopard concept}

Single layer TGEMs are tested in a TCPD-like [9] chamber to avoid microstructure overlapping from the usually used several MPGD stages. Light coming from the "top" of the detector first traverses a quartz window, then the optically $97 \%$ transparent wire-grid cathode and arrives to the top of the TGEM in study. Underneath the TGEM a CCC $[10,11]$ type wire chamber serves as the high gain post amplification stage right above the grounded baseplate. Signals are taken from the connected sense wires.

Hence in RICH detectors the UV dominated Cherenkov light emits electrons from the CsI surface, due to technical difficulties we simulate this using gold surface and a pulsed UV LED [12] (with peak wavelength of $245 \mathrm{~nm}$ ). Due to the low quantum efficiencies moderate UV intensity is used, tuned to have only several percent of photoelectrons (PE) per event to ensure that those were single PE signals.

After traversing an exchangeable pinhole, the light is focused with a quartz lens onto the upper surface of the TGEM. Duration and frequency of the UV pulses are set to keep good timing for signals and exploit the bandwidths of the DAQ system. High resolution is reached via focused spot size with $70 \mu \mathrm{m}$ FWHM to study TGEM structures [13]; for GEM foil examination $\sim 20 \mu \mathrm{m}$ had to be achieved.

The optical system is fixed to a three dimensional positioning stage right above the chamber. This way the light-spot can be moved throughout the TGEM surface, while with the third axis one can finetune the focusing. 
In each point several thousands of events are recorded, where from the single PE spectrum the light yield and the gas gain can be measured separately. Using the method above two dimensional maps of efficiency and amplification were measured with excellent granularity.

With the support of the RD51 Common Project the formerly used system [13] has been seriously upgraded, detailed description of the new DAQ can be seen below.

\section{Leopard RPi DAQ}

For high resolution images spatial distance of measurement points should be in the order of tenth of the periodic distance of the structure. Single PE signal needs low photoelectron emission probability per event, however roughly several hundreds of signal events are needed for a spectrum, leading tens of thousands of triggers in each point. Fulfilling the former requirements one should record billions of events in a reasonable time makes the speed of the DAQ crucial.

While the data acquisition system should take care of the fast event-by-event measurements, it should control the adequate synchronized moving of the positioning stage, store all the valuable data, and eventually set additional chamber parameters as well.

To realize all these functions we have designed a special PCB board (LRD Board) and coupled it to a RaspberryPi [14] microcomputer via its GPIO pins. A photo of the device can be seen on Fig. 2. left. The schematics of the whole Leopard setup is shown on Fig. 2. right, while a photo is shown on Fig. 1.

The board receives an asynchronous trigger and its delayed rising edge serves as selection time for a 12-bit ADC (LTC1415 [15]) to measure the signal from the chamber (after the tunable postamplification stages). The ADC is read out directly with the RaspberryPi using the GPIO pins.

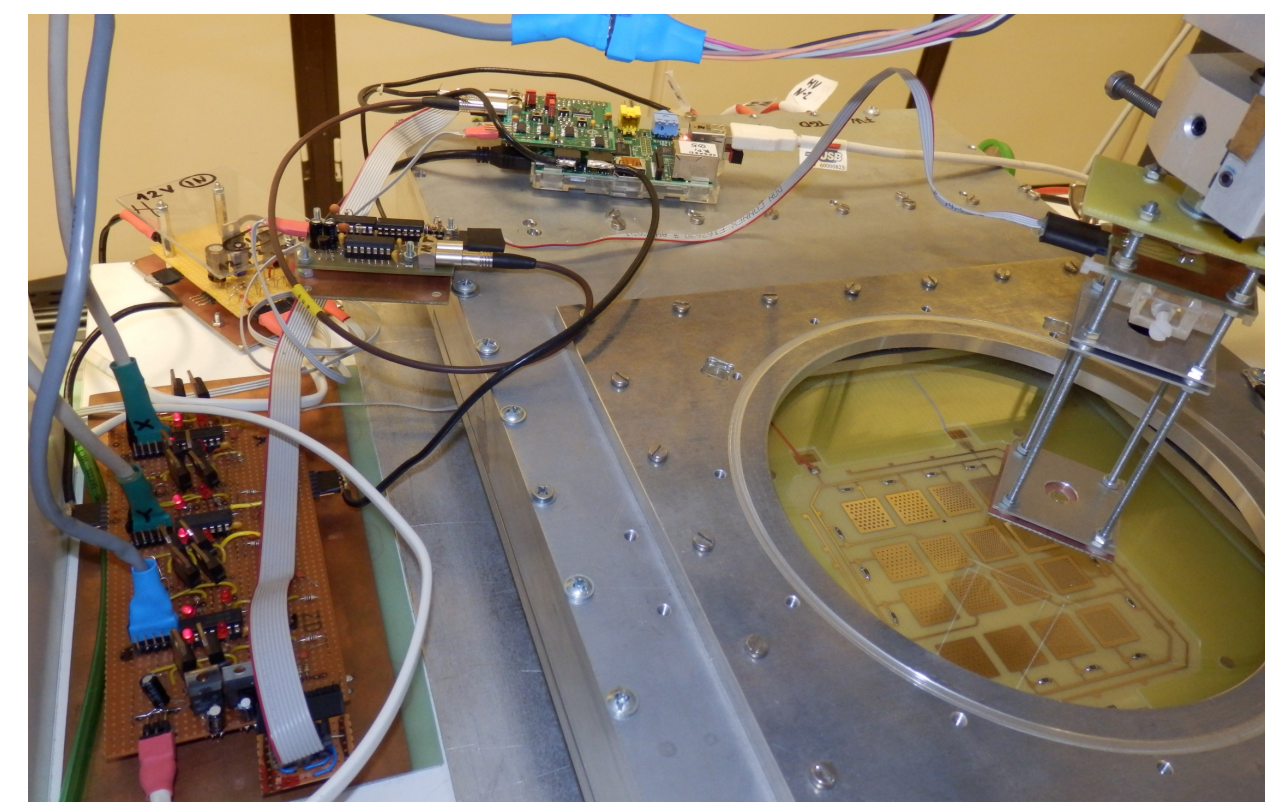

Figure 1: Full Leopard setup, on the right side above the chamber there is the optical stand attached to the positioning stage. On the other side there are the used electrical equipments: positioning-motor control, low voltage stabilizer and distributor, LED pulser, and the RPi based DAQ. 

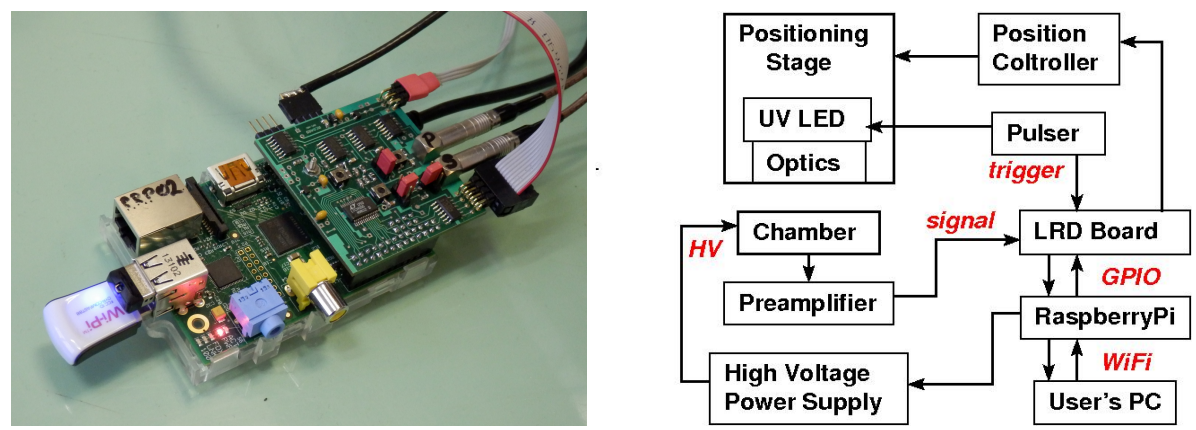

Figure 2: (left) The Leopard data acquisition board on the RaspberryPi. (right) The schematics of the Leopard setup.

In case a trigger arrives during the "busy" period it flips a one-bit flag (missed signal) which is read out in the next event; thus allowing the possibility to make digital signal processing at software level.

An event rate of more than $100 \mathrm{kHz}$ has been achieved with the present system. On Fig. 3. left there is an oscilloscope screenshot showing the triggers and the chamber signals with one photoelectron event besides the several pedestal signals.

The DAQ software runs on the RaspberryPi under a standard Debian clone Raspbian linux system. The source is written in $C / C++$ to make it fast and flexible at once. The program accepts command line inputs and settings file type inputs thus it is convenient to handle it remotely. Standard position and voltage scanning programs can be defined and be used, and even special position or voltage lists can be loaded as well.

The DAQ program is accessible by a graphical user interface too, running on a distant machine and communicating through standard internet link (for the RaspberryPi WiFi is used to eliminate ground loops from the ethernet cables). A screenshot on the "Leopard RaspberryPi DAQ GUI (LRDG)" can be seen on Fig. 3.
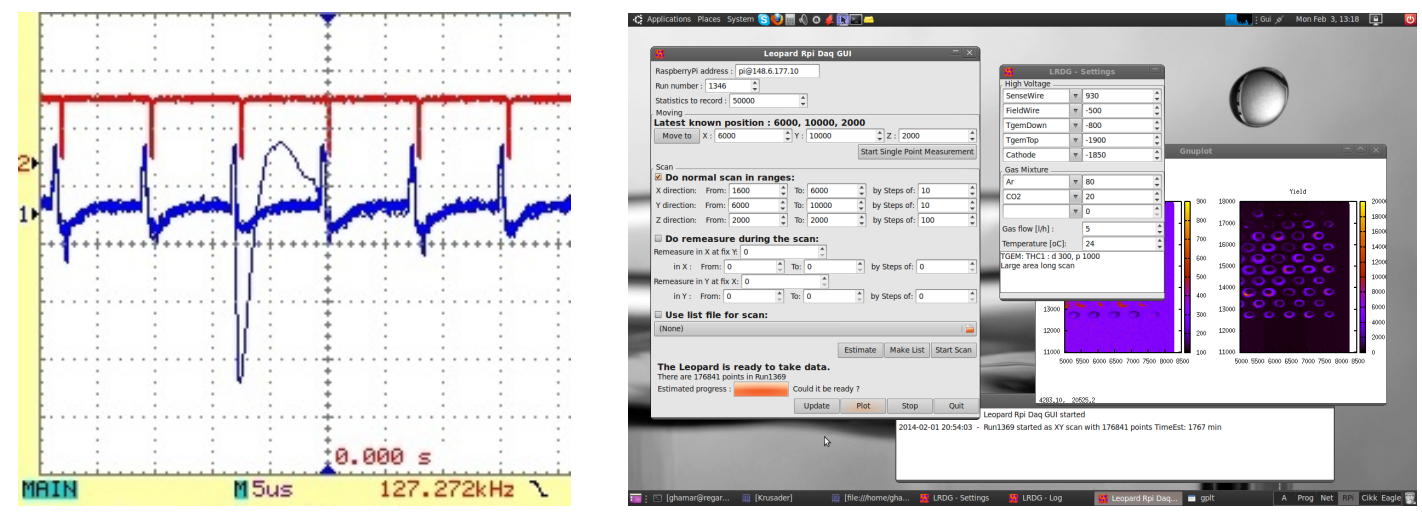

Figure 3: (left) Oscilloscope picture of some overlapped events with low photoelectron probability. Time scale is $5 \mu s$ per division. Triggers (red) are coming with $127 \mathrm{kHz}$ frequency from the UV LED pulser. On the detector signals (blue) the usual pedestal and one PE event can be seen. (right) Screenshot of the graphical interface (LRDG) for the Leopard system. All the measurement settings, e-logbook, monitoring, and $\log$ windows are shown. 


\section{Setting up the measurements}

From the dimensions of the chamber and the optical setup, and the refraction index of the quartz lens (in UV) one can set the focus onto the top surface of the TGEM within the several $\mathrm{mm}$ range. However fine tuning of this focal plane settings is necessary, and can be done on the sub-millimeter scale via the recorded data itself.

Map of the number of detected photoelectrons with different focal plane heights can be seen on Fig. 4. : left for TGEM, right for GEM foil. In all cases the sharpest picture should refer to the best focal settings. After the rough scan $(\delta Z=1.0 \mathrm{~mm})$ an other one with ten times finer steps around the focus used to be done.

While the method provides the best focused image, the spot size (and resolution) cannot be extracted from it, because it cannot be separated from the microstructure effects. Resolution power was measured with known binary-like structures using wires as shaders, see [13].

Long term stability is important for large statistics large area scans. Including into the scan a regular remeasuring of a certain region of the map stability of gain, light yield, and positioning precision can be evaluated; and data can be corrected offline for these effects if needed.
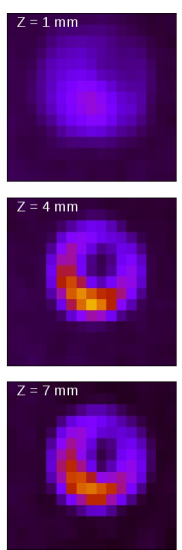
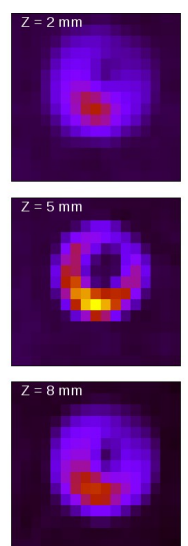
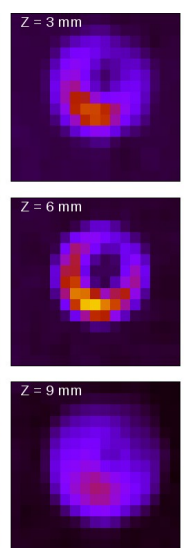

Focal Scan Maps with $\mathrm{dZ}=1.0 \mathrm{~mm}$
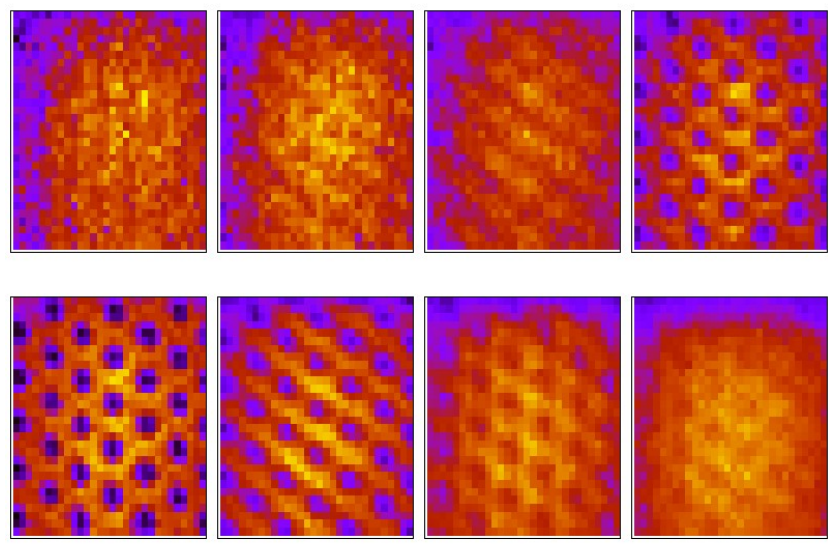

Figure 4: (left) Rough focal scan with maps on single hole (in a TGEM with $2 \mathrm{~mm}$ pitch) with $1 \mathrm{~mm}$ vertical steps. (right) Rough focal scan with maps on a GEM foil with $1 \mathrm{~mm}$ vertical steps.

\section{Results on TGEM microstructure}

Measurements were taken on a gold plated TGEM with $300 \mu \mathrm{m}$ hole diameter, $800 \mu \mathrm{m}$ pitch, $60 \mu \mathrm{m}$ rim, and $400 \mu \mathrm{m}$ thickness; produced at CERN in 2009. Gain of the TGEM was 7, and wire gain was several thousands to fit signals into the range of the used preamplifier. The filling gas mixture was $\mathrm{Ar}: \mathrm{CO}_{2}$ 80:20.

The detection efficiency is proportional to the integral of the spectrum from a non-zero pulse height (5 RMS of the noise). The holes of the TGEM are naturally present as dark spots in a hexagonal grid, with practically zero yield. Additional dark regions appear along the symmetry lines of two holes, and at the critical/symmetry points of three holes, where the field lines cannot 
go into any of the holes due to symmetry reasons. This results ring-like effective structures around the rim. An efficiency map can be seen on Fig. 5. left. Note that the total efficiency changes from hole to hole, and not circularly symmetric around the hole. These type of efficiency maps gave the project the name: Leopard.

The thickness of the ring-like structures depend on the cathode field [13] (and the filling gas) and on the geometrical parameters. Detailed studies of these questions and the corresponding optimizations are underway.

From the single photo-electron spectra the gain can be calculated for each measurement point. On Fig. 5. right one can see, that the gain is locally uniform in the hexagonal collection region of a hole, independently on the emission point; thus let us define the hole-gain abstraction. The hole-gains vary from hole to hole within a factor of two for this piece of TGEM.
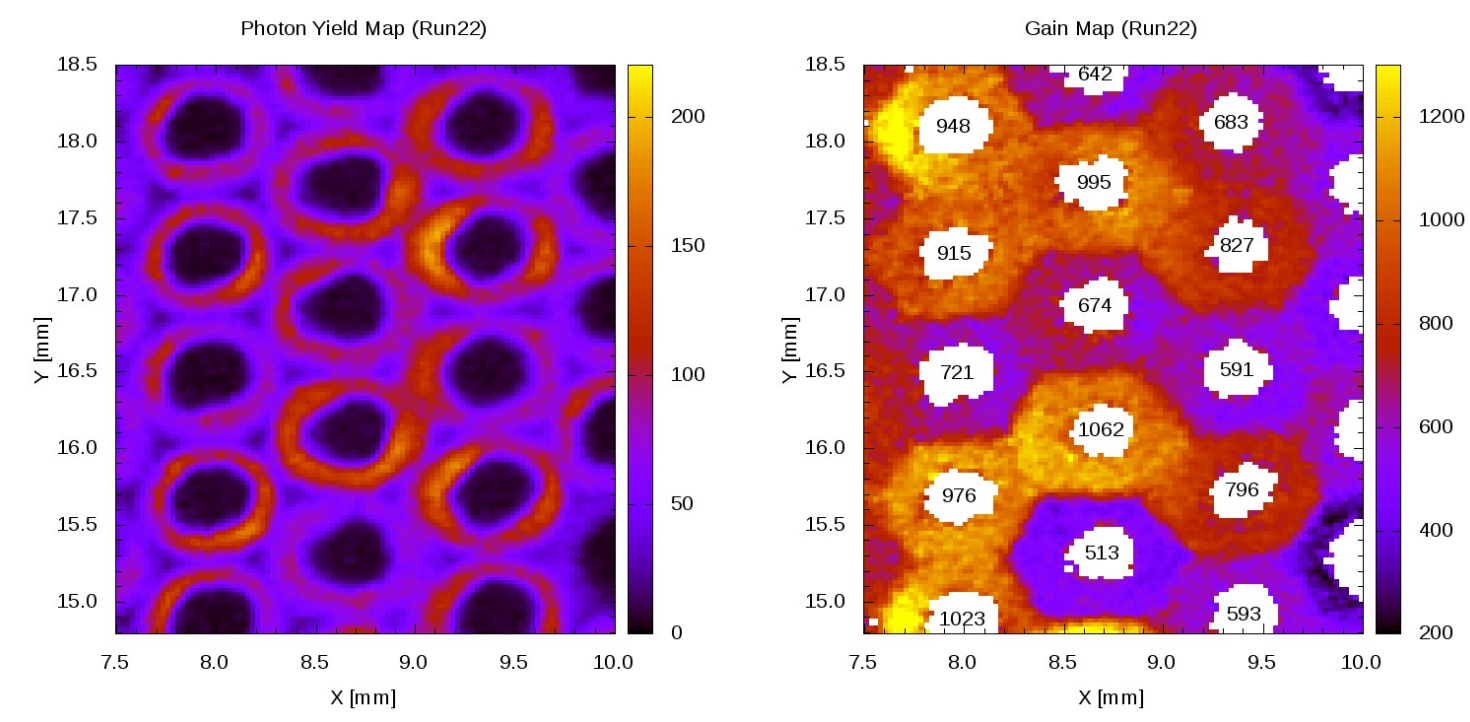

Figure 5: Maps of photoelectron yield and gain of a TGEM [13] with step of $25 \mu m$ with a $70 \mu m$ FWHM spot.

\section{Inspection of a standard GEM foil}

The new DAQ and upgrades of the optical stand made it possible to use pinholes of $30 \mu \mathrm{m}$ (spot size $\sim 20 \mu \mathrm{m}$ ), which is useable to examine standard GEM foils (50/70 $\mu \mathrm{m}$ hole with 140 $\mu m$ pitch).

For the measurements a gold plated GEM (produced at CERN in 2014) was used in pure methane with gain of 10 , while the wire gain was 6000 . Measurements were done with $10 \mu \mathrm{m}$ steps, the efficiency map can be seen on Fig. 6. for zero and $-800 \mathrm{~V} / \mathrm{cm}$ reversed drift fields on the same region. In the later case the symmetry points became visible as for TGEMs has been seen. 


\section{Scaled Yield at Different Drift Fields}

Zero Drift

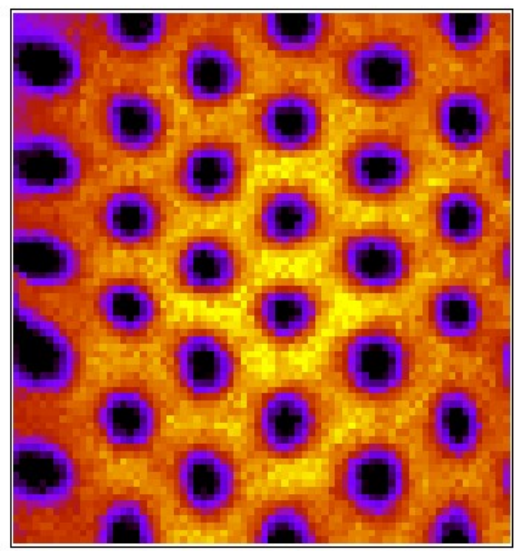

Large Reversed Drift

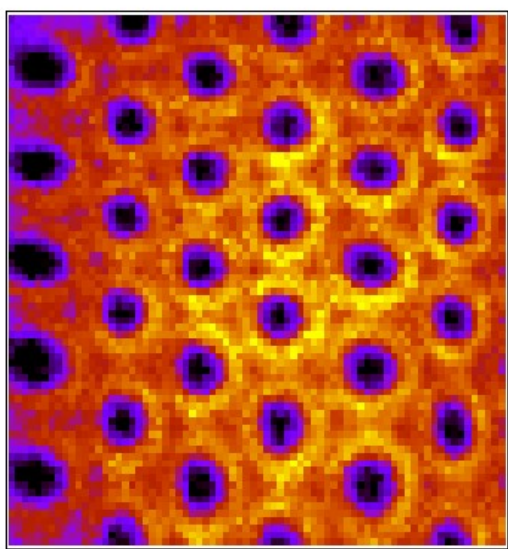

Figure 6: Efficiency map on a standard GEM foil, with pitch $0.14 \mathrm{~mm}$. Color coding refers to detected photoelectron yields.

\section{Discussion}

The Leopard system is capable to measure photoelectron yield and gain microstructure of MPGD based detectors, even for single layers (with coupling it to a wire chamber). Results can give us deeper knowledge on the relevant processes, and valuable input data for finetuning the simulations. The method can be naturally applied for optimization of TGEM (or other MPGD) geometry for better photon efficiency. Detailed studies of different TGEM geometries are foreseen to be tested within different gases for optimization for future RICH detectors. While the device can measure gain variation on the hole level it could be used for quality assurance checks coupled with optical inspection.

\section{Acknowledgement}

We would like to thank the assistance from the REGARD Group (Wigner RCP Budapest). This work has been support by the Hungarian "Lendület" LP2013-60 Programme of the HAS, and by the RD51 as Common Project. 


\section{References}

[1] F.Sauli,

Micro pattern gas detectors,

Nucl. Instr. and Meth. A 477 (2002) 1

[2] V.Peskov, M.Cortesi, R.Chechik, A.Breskin

Further evaluation of a THGEM UV-photon detector for RICH - comparison with MWPC

J.Instum. 5 (2010) P11004

[3] Z.Fraenkel et al.

A Hadron blind detector for the PHENIX experiment at RHIC

Nucl. Instr. and Meth. A 546 (2005) 466

[4] F.Sauli,

GEM: A new concept for electron amplification in gas detectors

Nucl. Instr. and Meth. A 386 (1997) 531

[5] A.Di.Mauro et al.,

The VHMPID RICH upgrade project for ALICE at LHC,

Nucl. Instr. and Meth. A 639 (2011) 274

[6] VHMPID/ALICE Collaboration,

A Very High Momentum Particle Identification Detector.

Eur. Phys. J. Plus 129 (2014) 91

[7] M. Alexeev et al.,

THGEM based photon detector for Cherenkov imaging applications,

Nucl. Instr. and Meth. A 617 (2010) 396

[8] A.Breskin et al.,

A Concise review on THGEM detectors.

Nucl. Instr. and Meth. A 598 (2009) 107

[9] G.Hamar, D.Varga,

TCPD, a TGEM based hybrid UV photon detector,

J. Instr. 8 (2013) C12038

[10] D.Varga, G.Hamar, G.Kiss,

Asymmetric multi-wire proportional chamber with reduced requirements to mechanical precision,

Nucl. Instr. and Meth. A 648 (2011) 163

[11] D.Varga, G.Hamar, G.Bencédi, G.Kiss,

Close Cathode Chamber: Low material budget MWPC,

Nucl. Instr. and Meth. A 698 (2013) 11

[12] Sensor Technology Inc., LED UVTOP240,

http://www.s-et.com/spec-sheets/240nm-with-images.pdf

[13] G.Hamar, D.Varga

High resolution surface scanning of Thick-GEM for single photo-electron detection

Nucl. Instr. and Meth. A 694 (2012) 16

[14] RaspberryPi,

http://www.raspberrypi.org/

[15] Linear Technology, LTC1415,

http://cds.linear.com/docs/en/datasheet/1415fs.pdf 